\title{
The generalized Boardman homomorphisms
}

\author{
Dominique Arlettaz* \\ Institut de mathématiques, \\ Université de Lausanne, \\ CH-1015 Lausanne, Switzerland
}

Received 4 November 2003; accepted 25 November 2003

\begin{abstract}
This paper provides universal upper bounds for the exponent of the kernel and of the cokernel of the classical Boardman homomorphism $b^{n}: \pi^{n}(X) \rightarrow H^{n}(X ; \mathbb{Z})$, from the cohomotopy groups to the ordinary integral cohomology groups of a spectrum $X$, and of its various generalizations $\pi^{n}(X) \rightarrow E^{n}(X), F^{n}(X) \rightarrow(E \wedge F)^{n}(X), F^{n}(X) \rightarrow H^{n}\left(X ; \pi_{0} F\right)$ and $F^{n}(X) \longrightarrow H^{n+t}\left(X ; \pi_{t} F\right)$ for other cohomology theories $E^{*}(-)$ and $F^{*}(-)$. These upper bounds do not depend on $X$ and are given in terms of the exponents of the stable homotopy groups of spheres and, for the last three homomorphisms, in terms of the order of the Postnikov invariants of the spectrum $F$.

(c) Central European Science Journals. All rights reserved.
\end{abstract}

Keywords: Boardman homomorphism, cohomotopy groups, generalized cohomology theories MSC (2000): Primary: $55 N 20,55$ Q 55; Secondary: 55 Q $45,55 S 45$

\section{Introduction and statement of the results}

The classical Boardman homomorphism for a spectrum $X$ is a homomorphism

$$
b^{n}: \pi^{n}(X) \longrightarrow H^{n}(X ; \mathbb{Z})
$$

between the cohomotopy groups and the ordinary integral cohomology groups of $X$ defined for all integers $n$ as follows:

$$
b^{n}: \pi^{n}(X)=\left[X, \Sigma^{n} S\right] \stackrel{\left(\Sigma^{n} i\right)_{*}}{\longrightarrow}\left[X, \Sigma^{n} H(\mathbb{Z})\right]=H^{n}(X ; \mathbb{Z})
$$

is induced by a generator $i: S \rightarrow H(\mathbb{Z})$ of $\pi_{0} H(\mathbb{Z}) \cong \mathbb{Z}$, where $S$ denotes as usual the sphere spectrum and $H(G)$ the Eilenberg-MacLane spectrum associated with the abelian group $G$.

\footnotetext{
*dominique.arlettaz@rect.unil.ch
} 
This short paper provides a couple of results establishing very general approximations of the size of the kernel and of the cokernel of the Boardman homomorphism and of its generalizations. Proofs are presented in the next section. Similar results have been obtained in [4] for the stable Hurewicz homomorphism.

When $X$ is an $(m-1)$-connected $d$-dimensional spectrum, $b^{n}$ is trivial if $n<m$ or $n>d$, an isomorphism if $n=d$ and an epimorphism if $n=d-1$. For $m \leq n \leq d-1$, one can easily show, using the Atiyah-Hirzebruch spectral sequence $H^{s}\left(X ; \pi_{-t} S\right) \Rightarrow \pi^{s+t}(X)$ that the kernel and the cokernel of $b^{n}$ are of finite exponent: the first goal of this paper is even to provide universal upper bounds for their exponent (see Theorem 1.2).

Definition 1.1. For $j \leq 0$ let $\rho_{j}=1$, and for $j \geq 1$ let $\rho_{j}$ be the exponent of the $j$-th homotopy group $\pi_{j} S$ of the sphere spectrum $S$.

Then define $\bar{\rho}_{i}=\prod_{j=1}^{i} \rho_{j}$ for $i \geq 1$.

Notice that a prime number $p$ divides $\bar{\rho}_{i}$ if and only if $p \leq \frac{i+3}{2}$.

The main theorem of the paper is the following:

Theorem 1.2. If $X$ is an $(m-1)$-connected spectrum of finite dimension $d$, the Boardman homomorphism $b^{n}: \pi^{n}(X) \rightarrow H^{n}(X ; \mathbb{Z})$ has the property that

(a) $\bar{\rho}_{d-n}$ ker $b^{n}=0$ for $m \leq n \leq d-1$ and

(b) $\bar{\rho}_{d-n-1} \operatorname{coker} b^{n}=0$ for $m \leq n \leq d-2$.

The second purpose of this note is to generalize this theorem in several ways. First, notice that it is possible to define a Boardman homomorphism by replacing the ordinary cohomology by other cohomology theories.

Definition 1.3. Let $E$ be a connective (i.e., (-1)-connected) ring spectrum with identity $i: S \rightarrow E$, and $E^{*}(-)$ the corresponding cohomology theory. For all integers $n$ and for any spectrum $X$, the E-Boardman homomorphism $\widehat{b}^{n}: \pi^{n}(X) \rightarrow E^{n}(X)$ is the homomorphism

$$
\widehat{b}^{n}: \pi^{n}(X)=\left[X, \Sigma^{n} S\right] \stackrel{\left(\Sigma^{n} i\right)_{*}}{\longrightarrow}\left[X, \Sigma^{n} E\right]=E^{n}(X)
$$

induced by $i$. Observe that if $X$ is of finite dimension $d$, then $\widehat{b}^{n}$ is trivial for $n>d$.

Remark. If $E=H(\mathbb{Z})$, the $H(\mathbb{Z})$-Boardman homomorphism is the classical Boardman homomorphism $b^{n}: \pi^{n}(X) \rightarrow H^{n}(X ; \mathbb{Z})$.

If $E$ and $X$ are finite spectra and if $X$ is finite dimensional, we are able to produce universal bounds for the exponent of the kernel of $\widehat{b}^{n}$.

Theorem 1.4. Let $E$ be a finite connective ring spectrum such that $\pi^{0}(E)$ is infinite cyclic, $E^{*}(-)$ the corresponding cohomology theory, and assume that $X$ is a finite $(m-1)$ - 
connected $d$-dimensional spectrum. Then the $E$-Boardman homomorphism $\widehat{b}^{n}: \pi^{n}(X) \rightarrow$ $E^{n}(X)$ satisfies

$$
\left(\rho_{m-n+1} \rho_{m-n+2} \cdots \rho_{d-n}\right) \operatorname{ker} \widehat{b}^{n}=0
$$

for any integer $n \leq d$. In particular, $\widehat{b}^{n}$ is injective if $n=d$ and $\bar{\rho}_{d-n}$ ker $\widehat{b}^{n}=0$ if $m \leq n \leq d-1$.

One can also extend Definition 1.3 and Theorem 1.4 as follows (see [6], p. 291).

Definition 1.5. Let $E$ be a connective ring spectrum with identity $i: S \rightarrow E, F$ any spectrum, and $F^{*}(-)$, respectively $(E \wedge F)^{*}(-)$, the cohomology theory associated with $F$, respectively with $E \wedge F$. For all integers $n$ and for any spectrum $X$, the $(F$ and $E \wedge F)$ Boardman homomorphism $\widehat{b}^{n}: F^{n}(X) \rightarrow(E \wedge F)^{n}(X)$ is the homomorphism

$$
\widehat{b}^{n}: F^{n}(X)=\left[X, \Sigma^{n}(S \wedge F)\right] \stackrel{\Sigma^{n}(i \wedge \mathrm{id})_{*}}{\longrightarrow}\left[X, \Sigma^{n}(E \wedge F)\right]=(E \wedge F)^{n}(X)
$$

induced by $i \wedge \mathrm{id}$, where id denotes the identity of $F$.

Definition 1.6. For a connective spectrum $F$, let $\rho_{j}(F)=1$ if $j \leq 0$ and, if $j \geq 1$, let us call $\rho_{j}(F)$ the order of the Postnikov invariant $k^{j+1}(F)$ of $F$ in the group $H^{j+1}(F[j-1]$; $\left.\pi_{j} F\right)$, where $F[j-1]$ is written for the $(j-1)$-st Postnikov section of $F$ (recall that $\rho_{j}(F)$ is finite by Theorem 1.5 of [2]).

Then define $\bar{\rho}_{i}(F)=\prod_{j=1}^{i} \rho_{j}(F)$ for $i \geq 1$.

Theorem 1.7. Let $E$ be a finite connective ring spectrum such that $\pi^{0}(E)$ is infinite cyclic, $F$ a connective spectrum, and $F^{*}(-)$, respectively $(E \wedge F)^{*}(-)$, the cohomology theory associated with $F$, respectively with $(E \wedge F)$. Furthermore, assume that $X$ is a finite $(m-1)$-connected $d$-dimensional spectrum. Then the $(F$ and $E \wedge F)$-Boardman homomorphism $\widehat{b}^{n}: F^{n}(X) \rightarrow(E \wedge F)^{n}(X)$ fulfills

$$
\left(\rho_{m-n+1}(F) \rho_{m-n+2}(F) \cdots \rho_{d-n}(F)\right) \operatorname{ker} \widehat{b}^{n}=0
$$

for any integer $n \leq d$. In particular, $\widehat{b}^{n}$ is injective if $n=d$ and $\bar{\rho}_{d-n}(F) \operatorname{ker} \widehat{b}^{n}=0$ if $m \leq n \leq d-1$.

Remark. In the case where $F$ is the sphere spectrum $S$, this gives the statement of Theorem 1.4, because $\rho_{j}(S)=\rho_{j}$ according to Theorem 1.3 of [4].

There is another way to generalize the definition of the Boardman homomorphism and the assertion of Theorem 1.2.

Definition 1.8. Let $F$ be a connective spectrum and $F^{*}(-)$ the corresponding cohomology theory. For all integers $n$ and for any spectrum $X$, the generalized $F$-Boardman homomorphism $\widetilde{b}^{n}: F^{n}(X) \rightarrow H^{n}\left(X ; \pi_{0} F\right)$ is the homomorphism 


$$
\widetilde{b^{n}}: F^{n}(X)=\left[X, \Sigma^{n} F\right] \stackrel{\left(\Sigma^{n} \alpha_{0}\right)_{*}}{\longrightarrow}\left[X, \Sigma^{n} H\left(\pi_{0} F\right)\right]=H^{n}\left(X ; \pi_{0} F\right)
$$

induced by the 0 -th Postnikov section $\alpha_{0}: F \rightarrow \underset{\sim}{H}\left(\pi_{0} F\right)$ of $F$ (i.e., such that $\left(\alpha_{0}\right)_{*}$ : $\pi_{0} F \rightarrow \pi_{0} H\left(\pi_{0} F\right)$ is an isomorphism). Notice that $\widetilde{b}^{n}$ is trivial if $n<m$ or $n>d$.

Remark. If $F=S$, the generalized $S$-Boardman homomorphism is exactly the classical Boardman homomorphism $b^{n}: \pi^{n}(X) \rightarrow H^{n}(X ; \mathbb{Z})$.

In this case, we get universal bounds for the exponent of the cokernel of the homomorphism $\widetilde{b}^{n}$, assuming that $X$ is finite dimensional.

Theorem 1.9. Let $F$ be a connective spectrum, $F^{*}(-)$ its associated cohomology theory, and consider an $(m-1)$-connected spectrum $X$ of finite dimension $d$. Then the generalized $F$-Boardman homomorphism $\tilde{b}^{n}: F^{n}(X) \rightarrow H^{n}\left(X ; \pi_{0} F\right)$ satisfies
(a) $\widetilde{b}^{n}$ is an isomorphism if $n=d$ and an epimorphism if $n=d-1$,
(b) $\bar{\rho}_{d-n-1}(F)$ coker $\widetilde{b}^{n}=0$ for $m \leq n \leq d-2$.

It is actually possible to extend Definition 1.8 by constructing a family of generalized $F$-Boardman homomorphisms as follows.

Definition 1.10. Let $F$ be a connective spectrum, $F^{*}(-)$ the corresponding cohomology theory, and $X$ an $(m-1)$-connected $d$-dimensional spectrum. Then, for any integer $n \leq d$ and any integer $t \geq 0$ such that $m-n \leq t \leq d-n$, there exists a generalized $F$-Boardman homomorphism

$$
\widetilde{b}^{n, t}: F^{n}(X) \longrightarrow H^{n+t}\left(X ; \pi_{t} F\right),
$$

which is defined as follows. The cohomological version of Lemma 4.2 of [2] implies that $F^{n}(X) \cong F(m-n, d-n]^{n}(X)$, where $F(m-n, d-n]$ is the spectrum with the property that $\pi_{i} F(m-n, d-n]=0$ if $i<m-n$ or $i>d-n$ and $\pi_{i} F \cong \pi_{i} F(m-n, d-n]$ if $m-n \leq i \leq d-n$ (see Section 4 of [2], Section 2.2 of [5], or Section 4 of [7]). Then, for any integer $t \geq 0$ with $m-n \leq t \leq d-n$, the homomorphism $\lambda_{d-n, t}: F(m-n, d-n] \rightarrow$ $\Sigma^{t} H\left(\pi_{t} F\right)$ introduced in Theorem 1.5 of [3] induces the homomorphism

$$
\widetilde{b}^{n, t}: F^{n}(X) \cong\left[X, \Sigma^{n} F(m-n, d-n]\right] \stackrel{\left(\Sigma^{n} \lambda_{d-n, t}\right)_{*}}{\longrightarrow}\left[X, \Sigma^{n+t} H\left(\pi_{t} F\right)\right]=H^{n+t}\left(X ; \pi_{t} F\right) .
$$

Observe that $\widetilde{b}^{n, 0}=\widetilde{b}^{n}$.

The exponent of the cokernel of these homomorphisms is also universally bounded.

Theorem 1.11. Let $F$ be a connective spectrum, $F^{*}(-)$ its associated cohomology theory, and $X$ an $(m-1)$-connected spectrum of finite dimension $d$. Then the generalized $F$-Boardman homomorphisms $\widetilde{b}^{n, t}: F^{n}(X) \rightarrow H^{n+t}\left(X ; \pi_{t} F\right)$ satisfy

$$
\left(\rho_{t}(F) \rho_{t+1}(F) \cdots \rho_{d-n}(F)\right) \operatorname{coker} \widetilde{b}^{n, t}=0
$$

for any $n \leq d$ and for any $t \geq 0$ with $m-n \leq t \leq d-n$. 


\section{Proofs}

Since Theorem 1.2 is based on the arguments involved in the proofs of Theorems 1.4 and 1.9 , we shall prove it at the end of this section.

Proof of Theorem 1.4. Since $E$ and $X$ are finite, we may conclude that

$$
E^{n}(X)=\left[X, \Sigma^{n} E\right] \cong\left[X ; \Sigma^{n}(S \wedge E)\right] \cong\left[E^{*} \wedge X, \Sigma^{n} S\right]=\pi^{n}\left(E^{*} \wedge X\right),
$$

where $E^{*}$ is the dual of $E$ (see [1], p. 195), and that the E-Boardman homomorphism $\widehat{b}^{n}$ is in fact

$$
\widehat{b}^{n}: \pi^{n}(X) \cong \pi^{n}(S \wedge X) \longrightarrow \pi^{n}\left(E^{*} \wedge X\right) \cong E^{n}(X),
$$

induced by $i^{*} \wedge \mathrm{id}$, where $i^{*}: E^{*} \rightarrow S$ is the dual of $i: S \rightarrow E$ and id the identity $X \rightarrow X$. Observe that, according to the cohomological version of Lemma 4.1 of [2], $\pi^{n}(X) \cong S[d-n]^{n}(X)$ since $X$ is of finite dimension $d$. The spectrum $E^{*} \wedge X$ is also finite dimensional: let us call $d^{\prime}$ its dimension (notice that $d^{\prime} \geq d$ ) and deduce similarly that $\pi^{n}\left(E^{*} \wedge X\right) \cong S\left[d^{\prime}-n\right]^{n}\left(E^{*} \wedge X\right)$. Then consider the commutative diagram

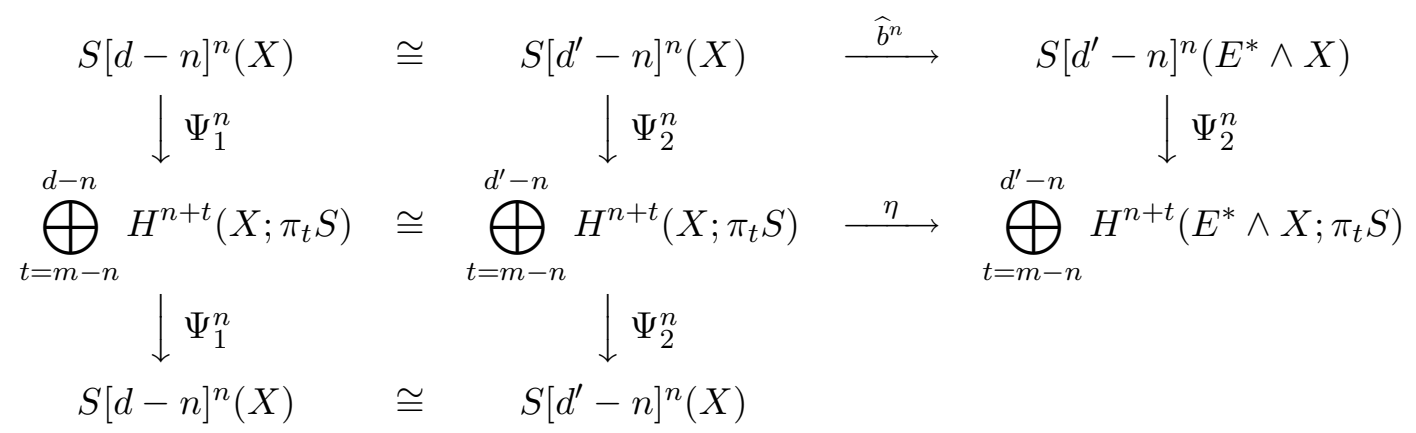

in which $\eta$ is also induced by $i^{*} \wedge \mathrm{id}: E^{*} \wedge X \rightarrow S \wedge X$, and where the vertical arrows are the homomorphisms introduced in Section 6 of [3] with the property that the composition $\Psi_{1}^{n} \Phi_{1}^{n}$ is multiplication by $\left(\rho_{m-n+1} \rho_{m-n+2} \cdots \rho_{d-n}\right)$. This follows from the fact that the order $\rho_{j}(S)$ of the $k$-invariant $k^{j+1}(S)$ of the sphere spectrum $S$ is equal to the exponent $\rho_{j}$ of $\pi_{j}(S)$ (see Theorem 1.3 of $[4]$ ). Since $\pi_{0} E^{*} \cong \pi^{0}(E) \cong \mathbb{Z}$, it turns out that $H_{j}(X ; \mathbb{Z}) \cong$ $H_{j}(X ; \mathbb{Z}) \otimes \pi_{0} E^{*}$ is a direct summand of $H_{j}\left(E^{*} \wedge X ; \mathbb{Z}\right)$ for any $j$ and thus that the homomorphism $\eta$ is split injective. Consequently, the commutativity of the diagram shows that the kernel of $\widehat{b}^{n}$ is contained in the kernel of the composition $\Psi_{1}^{n} \Phi_{1}^{n}$ : this produces the assertion of Theorem 1.4.

Proof of Theorem 1.7. As above, the finiteness of $E$ and $X$ implies that

$$
(E \wedge F)^{n}(X)=\left[X, \Sigma^{n}(E \wedge F)\right] \cong\left[E^{*} \wedge X, \Sigma^{n} F\right]=F^{n}\left(E^{*} \wedge X\right)
$$

and that

$$
\widehat{b}^{n}: F^{n}(X) \cong F^{n}(S \wedge X) \longrightarrow F^{n}\left(E^{*} \wedge X\right) \cong(E \wedge F)^{n}(X)
$$

is induced by $i^{*} \wedge$ id : $E^{*} \wedge X \rightarrow S \wedge X$. Then, let us use again the argument developed in the proof of Theorem 1.4 with the spectrum $F$ instead of $S$ and the integers $\rho_{j}(F)$ instead of $\rho_{j}$. 
The proof of Theorem 1.9 is based on the next lemma, the proof of which is analogous to that of Lemma 3.3 of [4].

Lemma. In the Atiyah-Hirzebruch spectral sequence $E_{2}^{s, t} \cong H^{s}\left(X ; \pi_{-t} F\right) \Rightarrow F^{s+t}(X)$ converging towards the $F$-cohomology of any $(m-1)$-connected $d$-dimensional spectrum $X$, the edge homomorphism $F^{n}(X) \rightarrow E_{\infty}^{n, 0} \hookrightarrow E_{2}^{n, 0} \cong H^{n}\left(X ; \pi_{0} F\right)$ is exactly the generalized $F$-Boardman homomorphism $\widetilde{b}^{n}$ for all integers $n$ such that $m \leq n \leq d$.

Proof of Theorem 1.9. Assertion (a) is trivial. Now, take $n$ such that $m \leq n \leq d-2$. Since $F$ is connective and $X$ is $d$-dimensional, the $E_{2}$-term of the Atiyah-Hirzebruch spectral sequence satisfies $E_{2}^{s, t} \cong H^{s}\left(X ; \pi_{-t} F\right)=0$ if $s>d$, and consequently $E_{\infty}^{n, 0}=$ $E_{d-n+1}^{n, 0}$. By the previous lemma, the image of $\widetilde{b}^{n}$ is $E_{d-n+1}^{n, 0}$, which is the subgroup of $E_{2}^{n, 0} \cong H^{n}\left(X ; \pi_{0} F\right)$ consisting of the kernel of the differentials $d_{r}^{n, 0}: E_{r}^{n, 0} \rightarrow E_{r}^{n+r, 1-r}$ for $2 \leq r \leq d-n$, and coker $\widetilde{b}^{n} \cong E_{2}^{n, 0} / E_{d-n+1}^{n, 0}$. However, it follows from Proposition 2.6 of [2] that $\rho_{r-1}(F) d_{r}^{n, 0}=0$. This implies that the product $\bar{\rho}_{d-n-1}(F)$ kills coker $\tilde{b}^{n}$.

Proof of Theorem 1.2. According to Theorem 1.3 of $[4], \rho_{j}(S)=\rho_{j}$ and Theorem 1.9 provides the second assertion of Theorem 1.2 For $X$ finite, the first assertion of Theorem 1.2 is exactly the statement of Theorem 1.4 for $E=H(\mathbb{Z})$. In the general case, the above lemma for the Atiyah-Hirzebruch spectral sequence $E_{2}^{s, t} \cong H^{s}\left(X ; \pi_{-t} S\right) \Rightarrow \pi^{s+t}(X)$ implies that the kernel of the classical Boardman homomorphism $b^{n}: \pi^{n}(X) \rightarrow H^{n}(X ; \mathbb{Z})$ is killed by the product of the exponents of the groups $E_{2}^{n-t, t}$ for $n-d \leq t \leq-1$, hence by the product $\bar{\rho}_{d-n}$.

Proof of Theorem 1.11. By definition of $\widetilde{b}^{n, t}$ and by Theorem 6.2 of [3], there is a homomorphism $\Theta^{n, t}: H^{n+t}\left(X ; \pi_{t} F\right) \rightarrow F^{n}(X)$ such that the composition

$$
H^{n+t}\left(X ; \pi_{t} F\right) \stackrel{\Theta^{n, t}}{\longrightarrow} F^{n}(X) \stackrel{\widetilde{b}^{n, t}}{\longrightarrow} H^{n+t}\left(X ; \pi_{t} F\right)
$$

is multiplication by the product $\left(\rho_{t}(F) \rho_{t+1}(F) \cdots \rho_{d-n}(F)\right)$ on $H^{n+t}\left(X ; \pi_{t} F\right)$. This provides the assertion of Theorem 1.11 .

\section{References}

[1] J.F. Adams: Stable homotopy and generalised homology, The University of Chicago Press, Chicago, 1974.

[2] D. Arlettaz: "The order of the differentials in the Atiyah-Hirzebruch spectral sequence", K-Theory, Vol. 6, (1992), pp. 347-361.

[3] D. Arlettaz: "Exponents for extraordinary homology groups", Comment. Math. Helv., Vol. 68, (1993), pp. 653-672.

[4] D. Arlettaz: "The exponent of the homotopy groups of Moore spectra and the stable Hurewicz homomorphism", Canad. J. Math., Vol. 48, (1996), pp. 483-495.

[5] C.R.F. Maunder: "The spectral sequence of an extraordinary cohomology theory", Math. Proc. Cambridge Philos. Soc., Vol. 59, (1963), pp. 567-574 
[6] R.M. Switzer: Algebraic topology - homotopy and homology, Die Grundlehren der mathematischen Wissenschaften, 1975.

[7] J.W. Vick: "Poincaré duality and Postnikov factors", Rocky Mountain J. Math., Vol. 3, (1973), pp. 483-499 